\title{
Case Series Involving Obstetrics Experiences and Outcomes in Con- nective Tissue Disease Patients in UKMMC
}

\author{
Mohd Shahrir Mohamed Said ${ }^{1 *}$, Ahmad Syahir Mohamed Yazid ${ }^{1}$, Anusiya Chandra ${ }^{1}$, Jian Bin Eeu ${ }^{1}$, Nurhasifah Abd Aziz ${ }^{1}$, Fatin \\ Nadiah Ahmad Yazid ${ }^{1}$, Chew Kah Teik ${ }^{2}$, Sazliyana Syahrul Shaharir ${ }^{1}$
}

${ }^{1}$ Department of Medicine, Universiti Kebangsaan Malaysia Medical Centre, Malaysia

${ }^{2}$ Department of Obstetrics and Gynaecology, Universiti Kebangsaan Malaysia Medical Centre, Malaysia

"Corresponding author: Mohd Shahrir Mohamed Said, Department of Medicine, Universiti Kebangsaan Malaysia Medical Centre, Malaysia. Tel: +600148106300; Email: drobiwan@gmail.com

Citation: Said MSM, Yazid ASM, Chandra A, Eeu JB, Aziz NA, et al. (2018) Case Series Involving Obstetrics Experiences and Outcomes in Connective Tissue Disease Patients in UKMMC. J Family Med Prim Care Open Acc: JFOA-109. DOI: 10.29011/ JFOA-109. 100009

Received Date: 07 December, 2017; Accepted Date: 05 January, 2018; Published Date: 11 January, 2018

\begin{abstract}
Background and Aims: Connective tissue diseases (CTD) such as Systemic Lupus Erythematosus (SLE), Rheumatoid Arthritis (RA), Antiphospholipid Syndrome (APS) and others are likely to be worsening during pregnancy. There are poor pregnancy performances because of the abnormal immune system activity. The aim of the study was to observe the obstetrics experiences and the outcomes.

Materials and Methods: A retrospective study was done and a total of 58 cases of pregnancy were taken from year 20102015 in Universiti Kebangsaan Malaysia Medical Centre (UKMMC). Patients were selected from a list of pregnant women admitted to Patient Admission Centre (PAC) UKMMC diagnosed with CTD and the data was collected from their medical records.

Results: Fifty-eight pregnancies were observed in 44 women where there were fifty pregnancies (86\%) with SLE, 4 (7\%) pregnancies with RA and 4 (7\%) pregnancies with APS. There were 39 (67.2\%) pure CTD patients and 19 (32.8\%) CTD patients with comorbidities. There were $6(9.5 \%)$ pregnancies in active group and $52(82.5 \%)$ pregnancies in remission group. Among the 58 pregnancies, there were $33(57 \%)$ birth with no complication, 26 (27\%) fetal loss (miscarriage, intrauterine death), 8 $(14 \%)$ premature babies and $1(2 \%)$ neonatal death. Maternal complications were affected by comorbidities $(\mathrm{p}=0.007)$. Fetal outcomes were affected by anti-double stranded DNA $(\mathrm{p}=0.05)$. Disease activity was affected by pregnancy as the serology tests pre-pregnancy and during pregnancy showed significant association in Complement 3 ( $\mathrm{p}=0.021)$, Complement 4 $(\mathrm{p}=0.016)$ and Urine Protein Creatinine Index $(\mathrm{p}=0.001)$.
\end{abstract}

Conclusion: This study showed there is a significant association between pregnancy outcomes with antids DNA and comorbidities. There is significant association between pregnancy and Connective Tissue Disease Activity.

\section{Introduction}

Connective Tissue Diseases (CTD) include a multiplicity of chronic multisystem disorders with a high percentage of autoimmune conditions. This usually affects women frequently during their childbearing period. Every pregnancy in a patient with CTD should be regarded as high-risk pregnancy, as there are poor pregnancy performances because of the abnormal immune system activity. Therefore, it requires intensive monitoring and immediate treatment of clinical problems. For these reasons, for women suffering from CTD, who are pregnant or who intend to become pregnant, an interdisciplinary setting addressing all aspects of rheumatology, ob-gyn and neonatology needs to be provided [1].

The purpose of the study is to determine the effects of pregnancy to CTD activity and the pregnancy outcomes in CTD patients as justified by journal SLE in Pregnancyshowed that lupus flares are very much common in pregnancy [2]. It increases 


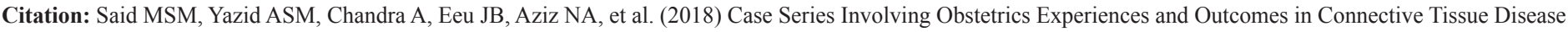
Patients in UKMMC. J Family Med Prim Care Open Acc: JFOA-109. DOI: 10.29011/JFOA-109. 100009

maternal morbidity, risk of premature delivery and fetal loss. Other than prematurity and fetal loss, neonatal lupus syndrome is another fetal complication. According to a retrospective study that was done in Peking Union Medical College Hospital in Beijing, there was a significant association between fetal and maternal outcomes with CTD activity during pregnancy [3].

\section{Methods}

We performed a retrospective study of 58 pregnancies of 44 women with CTD during a period of 5 years from January 2010-December 2015 managed in Department of Medicine and Department of Obstetrics and Gynaecology at Universiti Kebangsaan Malaysia Medical Centre (UKMMC).

UKMMC is a tertiary hospital located in Cheras, Kuala Lumpur. Kuala Lumpur is a federal capital city of Malaysia which primarily consists of a mix of Malays, Chinese and Indians, although there are many cultures in the city such as Eurasians, Kadazans, Ibans and indigenous peoples from East Malaysia and Peninsula Malaysia. Based on consensus done in 2010, the major ethnic consists of Malays/Bumiputera (45.9\%), Chinese (43.2\%), Indians (10.3\%) and others (1.6\%).

All the 44 women were identified from registration book between years 2010-2015 in Patient Admission Centre (PAC). With the name list, their medical records were requested from the department of medical record. Their information was retrieved from the medical records and OMS which included obstetrics history, medical history and laboratory investigation results (Erythrocyte Sedimentation Rate, Complement 3 and 4, Urine Protein Creatinine Index, Anticardiolipin and anti-double stranded DNA). The information was keyed in and analyzed using SPSS version 20.0 with standard statistical methods. Statistical significance was set at $\mathrm{P}<0.05$.

Autoimmune connective tissue diseases include Systemic Lupus Erythematous (SLE), Rheumatoid Arthritis (RA), AntiPhospholipid Syndrome (APS), Systemic Sclerosis and Sjorgen Syndrome and non-autoimmune connective tissue diseases include Marfan syndrome, Ehlers-Danlos Syndrome, Osteogenesis Imperfecta (OI) and Epidermolysis Bullosa.

In 1994, about $90 \%$ of SLE sufferers are women while about $10 \%$ are men and children and about $90 \%$ of women with SLE are in their childbearing years, within the range of 15 to 50 years old [4].

\section{Results}

\section{Patient Characteristics}

During study year conducted in 2010- 2015, there were 58 pregnancies in 44 patients with connective tissue disease. The ethnic separation was illustrated in (Table 1) Malay $(65.5 \%)$, Chinese (22.4\%), Indian (10.3\%) and others (1.7\%). About
22 patients were primigravida, while another 36 patients were multigravida. All the pregnancies were conceived naturally. The median age of the patients was 35.0 years and the median duration of disease was 6 years. The majority $(82.5 \%)$ of them were in clinical remission before pregnancy. The entire patients who were in clinical remission were on medication and they were compliance. The most common types of CTD found in our studies were SLE (86.2\%), APS (6.9\%) and RA (6.9\%), at which SLE was defined based on SLICC Classification Criteria (Table 2), [5], APS based on Sapporo Criteria (Table 3), [6], and RA was based on 2010 Rheumatoid Arthritis Classification Criteria (Table 4), [7]. Pure CTD was present in $(67.2 \%)$ of patients and about (32.8\%) patients of CTD with comorbidities. About 4 patients had positive anticardiolipin and 54 patients were having a negative anticardiolipin meanwhile for antids DNA, about 25 patients were positive and 33 patients were negative. Whereas, for the anti- LA, only 3 patients were tested to have positive results, and another 55 patients were tested negative. Most of them were on oral prednisolone with a mean dose of $5 \mathrm{mg}$ /day preconception and during conception.

\begin{tabular}{|c|c|}
\hline Age, mean (SD) & 35 years (4.36) \\
\hline Ethnicity & \\
\hline Malay & $65.50 \%$ \\
\hline Chinese & $22.40 \%$ \\
\hline Indian & $10.30 \%$ \\
\hline Others & $1.70 \%$ \\
\hline Duration of disease, mean (SD) & 10.18 years $(38.03)$ \\
\hline Comorbidities & \\
\hline Pure CTD & $67.20 \%$ \\
\hline CTD with comorbidities & $32.80 \%$ \\
\hline
\end{tabular}

Table 1: Demographics and clinical features of patients.

Requirements: $\geq 4$ criteria (at least 1 clinical and 1 laboratory criteria) OR biopsy-proven lupus nephritis with positive ANA or Anti-DNA.

\begin{tabular}{|c|c|}
\hline Clinical Criteria & Immunologic criteria \\
\hline 1. Acute cutaneous lupus * & 1. ANA \\
\hline 2. Chronic cutaneous lupus* & 2. Anti-DNA \\
\hline 3. Oral or nasal ulcer* & 3. Anti-SM \\
\hline 4. Non -scarring alopecia & 4. Antiphospholipid antibodies \\
\hline 5. Arthritis* & 5. Low complement (C3,C4,CH50) \\
\hline 6. Serositis* & $\begin{array}{c}\text { 6. Direct Coomb's test (do not } \\
\text { count in the presence in haemolytic } \\
\text { anemia) }\end{array}$ \\
\hline 7. Renal* & \\
\hline
\end{tabular}




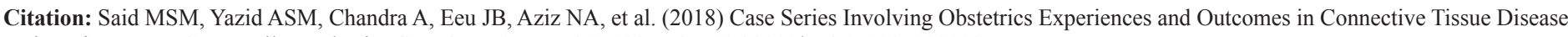
Patients in UKMMC. J Family Med Prim Care Open Acc: JFOA-109. DOI: 10.29011/JFOA-109. 100009

\begin{tabular}{|c|l|}
\hline 8. Neurologic $*$ & \\
\hline 9. Haemolytic Anemia & \\
\hline 10. Leukopenia* $^{*}$ & \\
\hline
\end{tabular}

$\begin{aligned} & \text { 11. Thrombocytopenia } \\ & \left(<100,000 / \mathrm{mm}^{3}\right)\end{aligned}$

\begin{tabular}{|c|}
\hline $\begin{array}{l}\text { Criteria for Antiphospholipid Syndrome (APS) } \\
\text { (Sydney revision of Sapporo criteria 2006) }\end{array}$ \\
\hline APS is present if at least 1 of the clinical criteria and 1 of the laboratory criteria that follow are met. \\
\hline Clinical criteria \\
\hline 1.Vascularthrombosis \\
\hline $\begin{array}{c}\text { One or more clinical episodes of arterial, venous or small-vessel thrombosis in any tissue or organ. Thrombosis must be confirmed by objectiv } \\
\text { validated criteria (unequivocal findings of appropriate imaging studies or histopathology). For histopathological confirmation, thrombosis shou } \\
\text { be present without significant evidence of inflammation in the vessel wall. }\end{array}$ \\
\hline 2. Pregnancy-related morbidity \\
\hline $\begin{array}{l}\text { a) One or more unexplained deaths of a morphogically normal fetus at or beyond the } 10 \text { week of gestation with normal fetal morphology } \\
\text { documented by ultrasonography or by direct examination of the fetus, (or) }\end{array}$ \\
\hline $\begin{array}{l}\text { b) One or more premature births of a morphologically normal neonate before the } 34 \text { th week of gestation because of (i) eclampsia or sever } \\
\text { preeclampsia described according to standard definitions (or) (ii) recognized features of placentalinsuffiency (or) }\end{array}$ \\
\hline $\begin{array}{l}\text { c) Three or more unexplained consecutive spontaneous abortions before the 10th week of gestation, with maternal anatomical or hormonal } \\
\text { abnormalities and paternal and maternal chromosomal causes excluded. }\end{array}$ \\
\hline Laboratory Criteria \\
\hline All laboratory criteria should be present one 2 or more occasions, at least 12 weeks apart. \\
\hline 1. LA present in plasma, detected according to the guidelines of the ISTH (Scientific Subcommitee on LAs/ phospholipid dependent antibodies \\
\hline $\begin{array}{l}\text { 2. ACL antibody of IGG and/or IGM isotope in serum or plasma, present in medium or high titer ( }>40 \text { GPL or MPL, or }>99^{\text {th }} \text { percentile), } \\
\text { measured by a standardized ELISA. }\end{array}$ \\
\hline $\begin{array}{l}\text { 3. Anti-B2GP! Of IGG and/or IGM isotope in serum or plasma (in titer }>99^{\text {th }} \text { percentile), measured by a standardized ELISA, according to } \\
\text { recommended procedures. }\end{array}$ \\
\hline
\end{tabular}

Table 3: Criteria for Antiphospholipid Syndrome. 


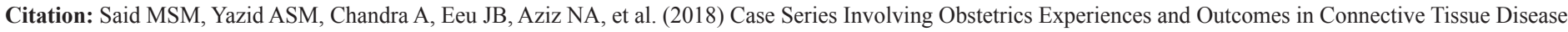
Patients in UKMMC. J Family Med Prim Care Open Acc: JFOA-109. DOI: 10.29011/JFOA-109. 100009

\begin{tabular}{|c|c|}
\hline & SCORE \\
\hline \multicolumn{2}{|l|}{ Target population (who should be tested?): Patients who: } \\
\hline \multicolumn{2}{|l|}{ 1. Have at least 1 joint with definite clinical synovitis (swelling) } \\
\hline \multicolumn{2}{|l|}{ 2. With the synovitis not better explained by another disease } \\
\hline \multicolumn{2}{|c|}{$\begin{array}{c}\text { Classification criteria for RA (score-based algorithm: add score of categories A-D, a score of } \geq 6 / 10 \text { is needed for } \\
\text { classification of a patient as having definite RA) }\end{array}$} \\
\hline \multicolumn{2}{|l|}{ A. Joint Involvement } \\
\hline 1 large joint & 0 \\
\hline 2-10 large joints & 1 \\
\hline 1-3 small joints (with/without involvement of large joints) & 2 \\
\hline 4-10 small joints (with/without involvement of large joints) & 3 \\
\hline$>10$ joints (at least 1 small joint) & 5 \\
\hline \multicolumn{2}{|l|}{ B. Serology (at least 1 test result is needed for classification } \\
\hline Negative RF and negative ACPA & 0 \\
\hline Low-positive RF or low-positive ACPA & 2 \\
\hline High-positive RF or high-positive ACPA & 3 \\
\hline \multicolumn{2}{|l|}{ C. Acute-phase reactant (at least 1 test result is needed for classification) } \\
\hline Normal CRP and normal ESR & 0 \\
\hline Abnormal CRP or abnormal ESR & 1 \\
\hline \multicolumn{2}{|l|}{ D. Duration of symptoms } \\
\hline$<6$ weeks & 0 \\
\hline$\geq 6$ weeks & 1 \\
\hline
\end{tabular}

Table 4: 2010 ACR/EULAR criteria for Rheumatoid Arthritis.

\section{Effect of Pregnancy on CTD Disease Activity}

There were eleven episodes of relapse (19\%) in the 58pregnancies, and four $(6.9 \%)$ occurred in second trimester and seven $(12.1 \%)$ in third trimester $(\mathrm{p}>0.05)$. None occurred in the first trimester and 47 pregnancies $(81 \%)$ had no relapse at all. ESR, C3, C4 and UPCI pre-pregnancy results were taken at least 4 months before pregnancy and the earliest laboratory investigations done during pregnancy were taken. The results were compared to see the significant effect of pregnancy on CTD disease activity. C3, C4 and UPCI were significantly affected by pregnancy as changes in C3 showed $(\mathrm{p}<0.021), \mathrm{C} 4(\mathrm{p}<0.016)$ and UPCI $(\mathrm{p}<0.001)$.

\section{Effect of CTD on Maternal Complications}

Out of 58 cases of pregnancies, only 16 cases of pregnancies which was from SLE patients that gave effect on maternal complications whereas RA and APS both have no maternal complication ( $p>0.05)$. Majority of the SLE patients $(72.4 \%)$ have no complication and followed by $6(10.3 \%)$ pregnancies with preeclampsia, 4(6.9\%) pre-labour rupture of membrane (PROM), 2 $(3.4 \%)$ of eclampsia, preterm labour and others (placenta previa type 2 and severe chorioamnionitis) $(\mathrm{p}>0.05)$. Comorbidity showed significant association with maternal complications where there were $39(67.2 \%)$ of pure CTD and $19(32.8 \%)$ of CTD with comorbidities $(\mathrm{p}>0.05)$. Variables such as nephritis, remission of disease and relapse of disease have no significant effect on maternal complication. This was because there were more SLE patients without nephritis than with nephritis and there was more remission of disease and less relapse of disease.

\section{Effects of CTD on Fetal Outcome}

Only SLE patients suffered foetal complications from our research. There were 16 foetal losses out of the 58 pregnancies which consist of $12(20.7 \%)$ miscarriages, 3(5.2\%) intrauterine death and $1(1.7 \%)$ severe post-partum haemorrhage. Termination of pregnancy was given medically $(\mathrm{p}>0.05)$. There were $8(12.7 \%)$ preterm births and $1(1.6 \%)$ live birth complicated by neonatal death $(\mathrm{p}>0.05)$. The median weight of the live births was $2.41 \mathrm{~kg}$ and they have median Apgar score 8 at $1 \mathrm{~min}$ and 9 at $5 \mathrm{~min}(\mathrm{p}>0.05)$.

\section{Discussion}

This was a retrospective study involving 58 pregnancies in 44 women with CTD in UKMMC in a period of five years from 


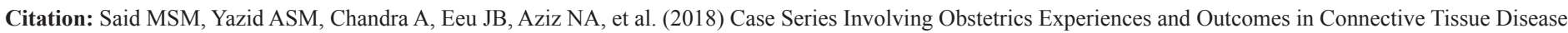
Patients in UKMMC. J Family Med Prim Care Open Acc: JFOA-109. DOI: 10.29011/JFOA-109. 100009

January 2010-December 2015. The subjects that we included in the study met our inclusion criteria which was pregnant women with CTD who went follow up and delivered in UKMMC. We excluded those who delivered in other hospitals. Thus, we collected data of 58 pregnancies where there were 50 patients with SLE, 4 patients with RA and 4 patients with APS based on their criteria mentioned in the results. The subjects' data collection was limited to 5 years because the duration of our study was only 1 year. We were expecting these were our study limitations.

There was a significant increase in C3, C4 and UPCI during pregnancy. They showed a significant association with pregnancy as C3 showed $(\mathrm{p}<0.021)$, C4 $(\mathrm{p}<0.016)$ and UPCI $(\mathrm{p}<0.001)$. $\mathrm{C} 3, \mathrm{C} 4$ and UPCI were indicators to monitor disease activity. Changes in these serology test values indicated that pregnancy had a good effect on disease activity thus allowing patients to have a safe pregnant. However, there were only few studies conducted on disease activity. Pre-conception counselling in UKMMC had shown an improvement in managing pregnant women with CTD. This was mentioned by Guilherme Ramires de Jesus et al. where patients who started a pregnancy in a stable remission period and continued on medications experienced fewer flares, which were mostly mild and generally well managed with a temporary increase in the prednisone dose [8].

As a result, there were only eleven episodes of relapse $(19 \%)$ in the 58 pregnancies, and four $(6.9 \%)$ occurred in second trimester and seven (12.1\%) in third trimester. Forty-seven pregnancies $(81 \%)$ had no relapse at all and this was supported by Guilherme Ramires de Jesus et al. study. Their study showed results of prospective, controlled observational studies show some discordance: some studies found that women are at increased risk of lupus flares when pregnant, while other studies found the rate of flares was unchanged as compared to no pregnant SLE patients [8]. This discrepancy may be explained by disease heterogeneity, the limited number of patients enrolled in SLE-pregnancy studies, the lack of homogeneous criteria for defining lupus flares, and the different SLE treatments used during pregnancy [8].

Out of 58 cases of pregnancies, only 16 cases of pregnancies which was from SLE patients that gave effect on maternal complications whereas RA and APS both have no maternal complication. Majority of the SLE patients (72.4\%) have no complication and followed by $6(10.3 \%)$ pregnancies with preeclampsia, 4(6.9\%) pre-labour rupture of membrane (PROM), 2 $(3.4 \%)$ of eclampsia, preterm labour and others (placenta previa type 2 and severe chorioamnionitis). In our study, we found that the worst maternal complication was pre-eclampsia as in a study conducted byCL the et al showed similar result [9].

Comorbidity showed significant association with maternal complications where there were $39(67.2 \%)$ of pure CTD and 19 $(32.8 \%)$ of CTD with comorbidities. Variables such as nephritis, remission of disease and relapse of disease have no significant effect on maternal complication. This was because there were more SLE patients without nephritis than with nephritis and there was more remission of disease and less relapse of disease.

Only SLE patients suffered foetal complications from our research. Results showed $33(56.9 \%)$ out of 58 pregnancies were live births and there were 16 foetal losses consist of $12(20.7 \%)$ miscarriages, $3(5.2 \%)$ intrauterine death and $1(1.7 \%)$ severe postpartum haemorrhage. There were $8(12.7 \%)$ preterm births and $1(1.6 \%)$ live birth complicated by neonatal death. Meanwhile, the study carried out byPark et al.Showedthat 51 of the 62 pregnancies $(82.3 \%)$ were live births and 11 pregnancies $(17.7 \%)$ resulted in fetal losses. Thirty-eight of the 51 livebirths $(74.5 \%)$ were full term, and 13 live births $(25.5 \%)$ were preterm. The fetal losses included three $(4.8 \%)$ spontaneous abortions, two (3.2\%) stillbirths and six $(9.7 \%)$ therapeutic abortions [10].

The median weight of the live births was $2.41 \mathrm{~kg}$ and they have median Apgar score 8 at $1 \mathrm{~min}$ and 9 at $5 \mathrm{~min}$. There are five types of medicines that safe to be used by SLE patients during pregnancy such as Hydroxychloroquine (HCQ), Immunosuppression, Nonsteroidal Anti-Inflammatory Drugs, low molecular weight heparin and corticosteroid. Most of the patients $(65.5 \%)$ using corticosteroid. 43.1\% patients using nonsteroidal anti-inflammatory during their pregnancy. $25.9 \%$ SLE patients using immunosuppression during pregnancy. 17.2\% SLE patients chose HCQ for their medicine. 15.5\% low molecular weight heparin were used by patients during pregnancy. However, according to G.Ramires et al, HCQ is the first choice of drug used during pregnancy due to no reported complications to foetus and neonates [8]. However, it is not commonly used in our study. $65.5 \%$ of SLE patients used corticosteroid in UKMMC. Corticosteroid can be used as anti-inflammatory properties in the short term and immunosuppressive actions in the long term. Corticosteroid can help prevent flares in clinically stable but serologically active patient. This is the reason that most of UKMMC doctors choose corticosteroid as drug for CTD pregnant women. The nonsteroidal anti-inflammatory drugs can be used for CTD pregnant women for relieving arthralgia or serositis in lowest dose and should be stopped after the 32th week due to high risk of fetal and maternal haemorrhage. This is the reason we had only $43.1 \%$ of pregnant women using nonsteroidal anti-inflammatory drug.

\begin{tabular}{|c|c|}
\hline hydroxychloroquine & $10(17.2)$ \\
\hline immunosuppression & $15(25.9)$ \\
\hline aspirin & $25(43.1)$ \\
\hline low molecular weight heparin & $9(15.5)$ \\
\hline steroid & $38(65.5)$ \\
\hline
\end{tabular}

Limitations of our study include: Firstly, the data was retrospective and in paper. Secondly, not all connective tissue 


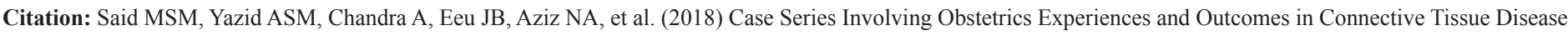
Patients in UKMMC. J Family Med Prim Care Open Acc: JFOA-109. DOI: 10.29011/JFOA-109. 100009

disease was represented. We failed to get data on non-SLE and non-Rheumatoid arthritis. Thirdly, compliance of these mothers taking their medication was not analysed.

\section{Acknowledgement}

We would like to thank the Dean of UKM Medical Faculty and Director of Chancellor Tuanku Muhriz Hospital for their cooperation in this study.

\section{References}

1. Saar P, Hermann W, Muller-Ladner U (2006) Connective tissue diseases and pregnancy. Rheumatology 45: 30-32.

2. Roy JS, Das PP, Datta A (2010) SLE in Pregnancy. Bangabandhu Sheikh Mujib Medical University Journal 3: 54-59.

3. Liu J, Zhao Y, Song Y, Zhang W, Bian X, et al. (2012) Pregnancy in women with systemic lupus erythematosus: a retrospective study of 111 pregnancies in Chinese women. J Matern Fetal Neonatal Med 25: 261-266.

4. Malaysia SLE Association: What is SLE ?.
5. Petri M1, Orbai AM, Alarcón GS, Gordon C, Merrill JT, (2012) et al. Derivation and Validation of Systemic Lupus International Collaborating Clinics Classification Criteria for Systemic Lupus Erythematosus. Arthritis Rheum 64: 2677-2686.

6. Miyakis S, Lockshin MD, Atsumi T, Branch DW, Brey RL, et al. (2006) International consensus statement on an update of the classification criteria for definite antiphospholipid syndrome (APS). J Thromb Haemos t4: 295-306.

7. Aletaha D, Neogi T, Silman AJ, Funovits J, Felson DT, et al. (2010) 2010 Rheumatoid arthritis classification criteria: an American College of Rheumatology/European League Against Rheumatism collaborative initiative. Arthritis Rheum 62: 2569-2581.

8. De Jesus GR, Mendoza-Pinto C, de Jesus NR, Dos Santos FC, Klumb EM, et al. (2015) Understanding and Managing Pregnancy in Patients with Lupus. Autoimmune Dis 2015: 943490.

9. Teh CL, Wong JS, Ngeh NK, Loh WL (2009) Systemic Lupus Erythematosus pregnancies: a case series from a tertiary East Malaysian hospital. Lupus 18: 278-282.

10. Park EJ, Jung H, Hwang J, Kim H, Lee J, et al. (2014) Pregnancy outcomes in patients with systemic lupus erythematosus: a retrospective review of 62 pregnancies at a single tertiary center in South Korea. International Int J Rheum Dis 17: 887-897. 